\title{
Dermatitis Radiation, CTCAE
}

National Cancer Institute

\section{Source}

National Cancer Institute. Dermatitis Radiation, CTCAE. NCI Thesaurus. Code C143405.

A finding of cutaneous inflammatory reaction occurring as a result of exposure to biologically effective levels of ionizing radiation. 